\title{
High-Performance Operating System Controlled Memory Compression
}

\author{
Lei Yang† \\ $\dagger$ (l-yang,dickrp)@ece.northwestern.edu \\ Northwestern University \\ Evanston, IL 60208
}

Haris Lekatsas

\author{
Robert P. Dick†

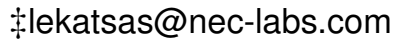 \\ NEC Laboratories America \\ Princeton, NJ 08540
}

\begin{abstract}
This article describes a new software-based on-line memory compression algorithm for embedded systems and presents a method of adaptively managing the uncompressed and compressed memory regions during application execution. The primary goal of this work is to save memory in disk-less embedded systems, resulting in greater functionality, smaller size, and lower overall cost, without modifying applications or hardware. In comparison with algorithms that are commonly used in on-line memory compression, our new algorithm has a comparable compression ratio but is twice as fast. The adaptive memory management scheme effectively responds to the predicted needs of applications and prevents on-line memory compression deadlock, permitting reliable and efficient compression for a wide range of applications. We have evaluated our technique on an embedded portable device and have found that the memory available to applications can be increased by $150 \%$, allowing the execution of applications with larger working data sets, or allowing existing applications to run with less physical memory.
\end{abstract}

Categories and Subject Descriptors: C.3 [Special-Purpose and Application-Based Systems]: Real-time and embedded systems

General Terms: Algorithms, Management, Performance

Keywords: Virtual memory, Compression

\section{Introduction}

Every year, embedded system designers pack more functionality into less space, while meeting tight performance and power consumption constraints. Software running on some embedded devices, such as cellular phones and PDAs, is becoming increasingly complicated, requiring faster processors and more memory to execute. In this work, we propose a method of increasing functionality without changes to hardware or applications by making better use of physical memory via on-line memory compression.

Our existing memory compression framework, CRAMES [1], takes advantage of an operating system's (OS's) virtual memory infrastructure by storing swapped-out pages in compressed format. It dynamically adjusts the size of the compressed RAM area, protecting applications capable of running without it from performance or energy consumption penalties. This memory compression technique has been implemented as a loadable module for the Linux kernel and evaluated on a battery-powered embedded system. Experimental results indicate that it is capable of doubling the amount of RAM available to applications. When used for this purpose, it has little or no impact on the execution times and energy consumptions of applications capable of running without CRAMES.

Although this version of CRAMES that used existing compression algorithms greatly increased usable memory for a wide range of applications, using it to cut physical memory to $40 \%$ increased application execution time by $29 \%$, in the worst case. This conflicted with our goal of dramatically increasing the memory avail-

\footnotetext{
This work is supported in part by NEC Laboratories America and in part by the NSF
} under award CNS-0347941.

Permission to make digital or hard copies of all or part of this work for personal or classroom use is granted without fee provided that copies are not made or distributed for profit or commercial advantage and that copies bear this notice and the full citation on the first page. To copy otherwise, to republish, to post on servers or to redistribute to lists, requires prior specific permission and/or a fee.

DAC 2006, July 24-28, 2006, San Francisco, California, USA.

Copyright 2006 ACM 1-59593-381-6/06/0007 ...\$5.00. able to applications without changing applications, without changing hardware, and without significant performance degradation, for all realistic embedded applications. In this article, we describe two techniques to further improve the performance of on-line softwarebased memory compression.

We present a very fast, high-quality compression algorithm for working data set pages. This algorithm, named pattern-based partial match (PBPM), explores frequent patterns that occur within each word of memory and takes advantage of the similarities among words by keeping a small two-way, hashed, set associative dictionary that is managed with a least-recently used (LRU) replacement policy. PBPM has a compression ratio that is competitive with the best compression algorithms of the Lempel-Ziv family [2] while exhibiting lower run-time and memory overhead.

In addition, we present an adaptive compressed memory management technique that predictively allocates memory for compressed data. Experimental results show that our new pre-allocation method is able to further increase available memory to applications by up to $13 \%$ compared to the same system without pre-allocation.

\section{Related work}

A number of previous approaches incorporated compression into the memory hierarchy for different goals. Main memory compression techniques [3] insert a hardware compression/decompression unit between cache and RAM. Data is stored uncompressed in cache, and compressed on-the-fly when transferred to memory. Code compression techniques [4] store instructions in compressed format and decompress them during execution. Compression is usually done off-line and can be slow, while decompression is done during execution, usually by special hardware, and must be very fast.

Compressed caching $[5,6]$ introduces a software cache to the virtual memory system that uses part of the memory to store data in compressed format. Swap Compression [7,8] compresses swapped pages and stores them in a memory region that acts as a cache between memory and disk. The primary objective of both techniques is to improve system performance by decreasing the number of page faults that must be serviced by hard disks. Both techniques require a backing store, i.e., hard disks, when the compressed cache is filled up. Unlike hardware-based main memory compression techniques, neither compressed caching nor swap compression is able to compress code in memory.

IBM's MXT technology [3] used a hardware parallelized derivative of LZ77 [2]. Kjelso, et al. [9] designed X-Match, a hardwarebased dictionary coding algorithm. Wilson [6] proposed WKdm, a software-based dictionary coding algorithm. Rizzo [7] presented a software-based algorithm that compresses in-RAM data by exploiting the high frequency of zero-valued data.

\section{Overview of CRAMES}

This section briefly introduces the design and implementation of CRAMES to provide readers a context for the techniques presented in this paper. To increase available memory to embedded systems, CRAMES selectively compresses data pages when the working data sets of processes exceed physical RAM capacity. When data in a compressed page is later required by a process, CRAMES locates that page, decompresses it, and copies it back to the main memory working area, allowing the process to continue executing. In order to minimize performance and energy consumption impact, CRAMES takes advantage of the OS virtual memory swapping mechanism to decide which data pages to compress and when to perform compression and decompression. CRAMES requires an MMU. However, no other special-purpose hardware is required. 


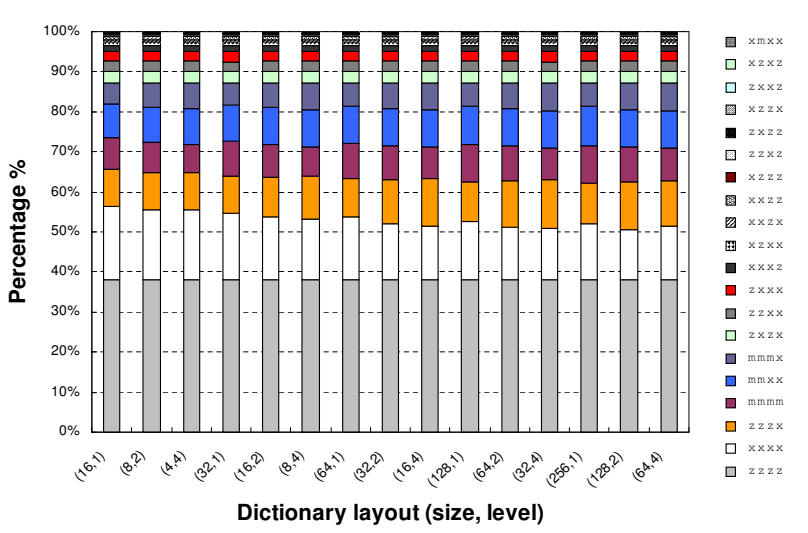

Figure 1: Frequent pattern histogram

To ensure good performance, the compression algorithm used in CRAMES must be highly efficient at both compression and decompression. It must also provide a good compression ratio, yet require little working memory. In addition, CRAMES must efficiently organize the compressed swap device to enable fast compressed page access and minimal memory waste. CRAMES dynamically adjusts the size of the compressed area during operation based on the amount of memory required so that applications capable of running without memory compression do not suffer performance or energy consumption penalties as a result of its use. In addition to data set compression, CRAMES also supports compression of arbitrary inRAM filesystems.

CRAMES has been implemented as a loadable Linux kernel module for maximum portability and modularity, however it can easily be ported to other modern OSs. The module was evaluated on a next generation smartphone prototype and a battery-powered PDA using well-known batch benchmarks as well as interactive applications with graphical user interfaces (GUIs). The results show that CRAMES is capable of dramatically increasing memory capacity with small performance and power consumption costs.

\section{Pattern-based partial match compression}

The original implementation of CRAMES used the LZO [10] algorithm to compress data in memory. LZO is significantly faster than many other general-purpose compression algorithms, e.g., LZW series algorithms, gzip, and bzip2. However, it is not designed for memory compression and therefore does not fully exploit the regularities of in-RAM data. In addition, LZO requires $64 \mathrm{~KB}$ of working memory, a significant overhead on many memory-constrained embedded systems. In summary, LZO is a general-purpose algorithm with good compression ratio and performance. However, better results are possible for the on-line memory compression application. In this paper, we analyze the regularities of in-RAM data and describe a new algorithm, named PBPM, that is extremely fast and well-suited for memory compression.

The PBPM algorithm is based on the observation that frequentlyencountered data patterns can be encoded with fewer bits to save space. Scanning through the input data a word ( 32 bits) at a time, PBPM exploits patterns that occur frequently within each word of memory and searches for complete and partial matches with dictionary entries to take advantage of the similarities among words. More specifically, (1) some patterns that are very frequent are encoded using special bit sequences that are much shorter than the original data, (2) patterns that do not fall into the above category and are found in a small dictionary are encoded using the index of their location in dictionary, and finally (3) patterns that do not frequently occur and cannot be found in the dictionary are stored in dictionary while the word contents are output.

\subsection{In-RAM data patterns}

Unlike general-purpose algorithms designed for text data, a specialpurpose algorithm designed for in-RAM data compression must fully exploit the regularities present in memory. In-RAM data frequently have certain patterns. For example, pages are usually zerofilled after being allocated. Therefore, runs of zeroes are commonly

\begin{tabular}{|l|l|l|c|c|}
\hline Code & Pattern & Output & Size (bits) & Frequency \\
\hline 00 & zzzz & 00 & 2 & $38.0 \%$ \\
01 & xxxx & $01 \mathrm{BBBB}$ & 34 & $21.6 \%$ \\
10 & mmmm & $10 \mathrm{bbbb}$ & 6 & $11.2 \%$ \\
1100 & $\mathrm{zzzx}$ & $1100 \mathrm{~B}$ & 12 & $9.3 \%$ \\
1101 & mmxx & $1101 \mathrm{bbbbBB}$ & 24 & $8.9 \%$ \\
1110 & mmmx & $1110 \mathrm{bbbbB}$ & 16 & $7.7 \%$ \\
1111 & zxzx & $1111 \mathrm{BB}$ & 20 & $3.1 \%$ \\
\hline
\end{tabular}

\section{Table 1: Pattern encoding in PBPM}

encountered during memory compression. Numerical values are often small enough to be stored in 4,8 , or 16 bits, but are normally stored in full 32-bit words. Furthermore, numerical values tend to be similar to other values in nearby locations. Likewise, pointers often point to adjacent objects in memory, or are similar to other pointers in nearby locations.

In order to develop a reasonable set of frequent patterns, we experimented with a $64 \mathrm{MB}$ swap data file from a workstation running SuSE Linux 9.0. Various applications were executed to exhaust physical memory and trigger swapping. Figure 1 shows the relative frequencies of patterns we evaluated. Below we specify the conventions in describing the data and patterns, as well as the dictionary management scheme we considered.

We consider each 32-bit word (four bytes) as an input, and represent them with four symbols, each of which represents a byte. A ' $z$ ' represents a zero byte, an ' $x$ ' represents an arbitrary byte, and an ' $m$ ' represents a byte that matches with a dictionary entry. Following this convention, ' $z z z z$ ' indicates an all-zero word, while 'mmmx' indicates a partial match with one dictionary entry for which only the lowest byte differs.

To allow fast search and update operations, we maintain a hashmapped dictionary. More specifically, the third byte of a word is hash-mapped to a 256 entry hash table, the contents of which are random indices that are within the range of the dictionary. Based on this hash function, we only need to consider four match patterns: 'mmmm' (full match), 'mmmx' (highest three bytes match), 'mmxx' (highest two bytes match), and ' $x m x x$ ' (only the third byte matches). Note that neither the hash table nor the dictionary need be stored with the compressed data. The hash table is static and the dynamic dictionary is regenerated automatically during decompression.

We experimented with different dictionary sizes/layouts, e.g., 16entry direct-mapped and 32-entry two-way set associative, etc. A direct hash-mapped dictionary has the advantage of supporting fast search and update: only a single hashing operation and lookup are required per access. However, it has tightly limited memory. For each hash target, only the most recently observed word is remembered; the victim to be replaced is decided entirely by its hash target. In contrast, if a dictionary is maintained with move-to-front strategy, its LRU entry is selected as the victim. Unfortunately, searching in such a dictionary is slow. A set associative dictionary can enjoy the benefits of both LRU replacement and speed. When a search miss followed by a dictionary update occurs, the oldest of the dictionary entries sharing one hash target index is replaced.

As Figure 1 illustrates, zero words, 'zzzz', are the most frequent compressible pattern (38\%), followed by one byte positive signextended words 'zzzx' $(9.3 \%)$. 'zxzx' has a frequency of $2.8 \%$. Other zero-related patterns are infrequent. As the dictionary size increases, dictionary match (including partial match) frequencies do not increase much. While a set associative dictionary usually generates more matches than a direct hash-mapped dictionary with the same overall size, a four-way set associative dictionary works no better than a two-way set associative dictionary.

\subsection{The PBPM compression algorithm}

The PBPM compression and decompression algorithms are presented in Algorithm 1. PBPM maintains a small two-way set associative dictionary DICT[] of 16 recently-seen words. An incoming word can fully match a dictionary entry, or match only the highest three bytes or two bytes of a dictionary entry. These patterns occurred frequently during swap trace analysis. The patterns and coding schemes are summarized in Table 1 , which also reports the actual frequency of each pattern observed in our swap data file when other infrequent patterns are ignored. In Algorithm 1 and column 'Output' of Table 1, 'B' represents a byte and ' $b$ ' represents a bit. 

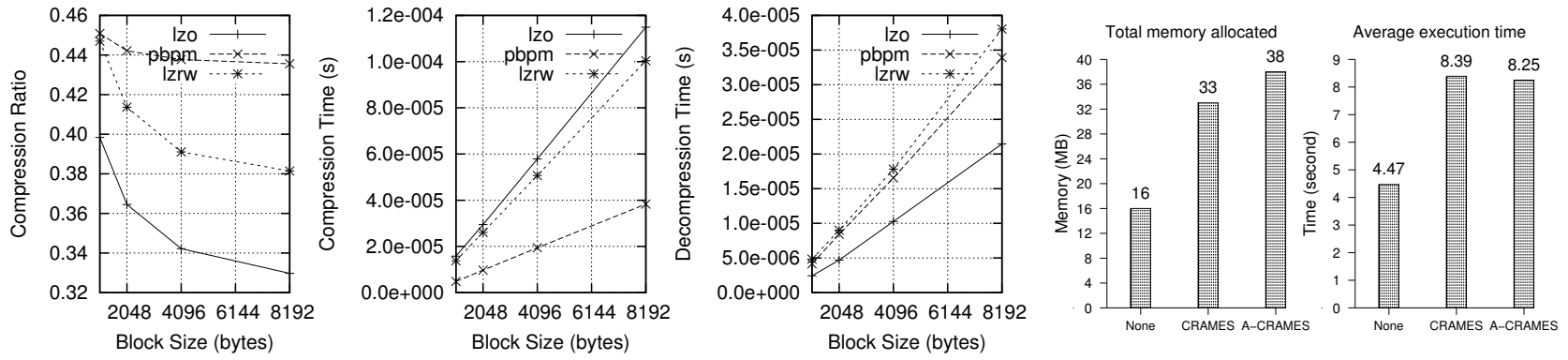

Figure 2: Compression ratios and speeds of PBPM, LZO, and LZRW

Figure 3: Performance of A-CRAMES

\begin{tabular}{|c|c|}
\hline \multicolumn{2}{|c|}{ Algorithm 1 PBPM (a) compression and (b) decompression } \\
\hline 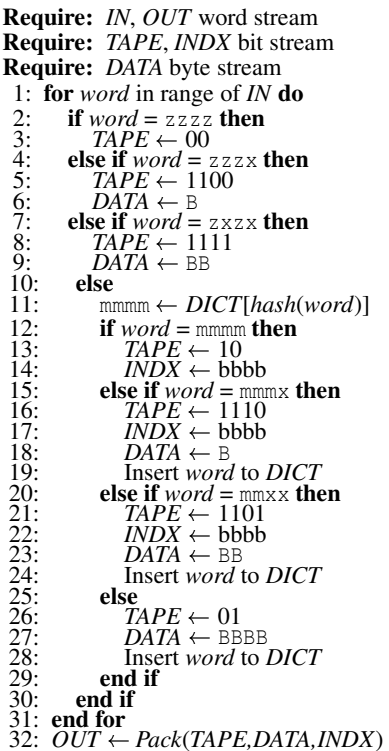 & 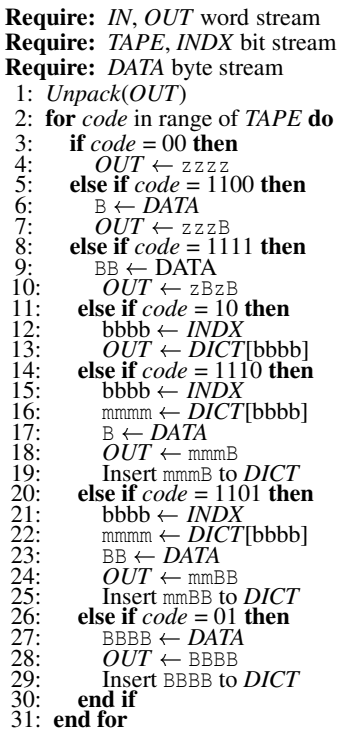 \\
\hline
\end{tabular}

\section{Adaptive compressed memory management}

As described in Section 3, CRAMES compresses the swapped-out data a page (4,096 bytes) at a time and stores them in a special compressed RAM device. Upon initialization, the compressed RAM device only requests a small memory chunk (usually $64 \mathrm{~KB}$ ) and requests additional memory chunks as system memory requirements grow. Therefore, the size of a compressed RAM device dynamically adjusts during operation, adapting to the data memory requirements of the currently running applications.

The above dynamic memory allocation strategy works well when the system is not under extreme memory pressure. However, it suffers from a performance penalty when the system is dangerously low on memory: applications and CRAMES compete for remaining physical memory and there is no guarantee that an allocation request will be satisfied. If physical memory is nearly exhausted, an application may be unable to allocate additional memory. If CRAMES were able to allocate even a page of physical memory for its compressed memory region, it would be able to swap out (on average) two pages, allowing the application to proceed. However, CRAMES is in contention with the application, resulting in a scenario we call on-line memory compression deadlock.

To avoid on-line memory compression deadlock, a compressed RAM device needs to be predictive during its requests for additional memory, i.e., it cannot wait until no existing chunks can allocate a fit slot for the incoming data. This subtle issue comprises a difference between our technique and compressed caching as well as swap compression, in which hard drives serve as backing store to which pages can be moved as soon as (or even earlier than) the compressed area is stuffed, so that the compressed memory is always available to applications.
We propose the following scheme to prevent on-line memory compression deadlock. CRAMES monitors the compressed area utilization and requests the allocation of a new memory chunk based on the saturation of current memory chunks. When the total amount of memory in the compressed area is above a predefined fill ratio, CRAMES requests a new chunk from kernel. This request may also be denied if the system memory is dangerously low. However, even if this first request is denied, subsequent invocations of CRAMES will generate additional requests. After low-memory conditions cause applications to swap out pages to the compressed RAM device, more memory will be available and the preemptive compressed RAM device allocation requests will finally be successful. We have experimented with different fill ratios and found that $7 / 8$ is sufficient to permit successful requests of compressed RAM device memory allocation in all tested applications. This ratio also results in little memory waste. Evaluation of this method on a portable embedded system is presented in Section 6.2.

\section{Evaluation}

In this section, we describe the evaluation methodology and results of the techniques proposed for high-performance on-line memory compression. More specifically, the following questions are experimentally evaluated:

1. Does PBPM provide a comparable compression ratio yet have even lower performance costs than existing algorithms?

2. Does adaptive memory management enable CRAMES to provide more memory to applications when system RAM is tightly constrained?

3. What is the overall performance of CRAMES using PBPM and adaptive memory management?

To evaluate the proposed techniques, we used a Sharp Zaurus SL5600 PDA. This battery-powered embedded system runs an embedded version of Linux, has a $400 \mathrm{MHz}$ Intel XScale PXA250 processor, $32 \mathrm{MB}$ of flash memory, and $32 \mathrm{MB}$ of RAM. In our current system configuration, $12 \mathrm{MB}$ of RAM are used for uncompressed, battery-backed filesystem storage and $20 \mathrm{MB}$ are available to kernel and user applications.

\subsection{Quality and speed of the PBPM algorithm}

We evaluated the compression ratio and speed of the PBPM algorithm compared to two other compression algorithms that have been used for on-line memory compression: LZO and LZRW. Figure 2 illustrates the compression ratios (compressed block size divided by original block size) and execution times of evaluated algorithms. For these comparisons, the source file for compression is the swap data file (divided into uniform-sized blocks) used to identify the frequent patterns in memory. The evaluation was performed on a Linux Workstation with a $2.40 \mathrm{GHz}$ Intel Pentium 4 processor. Note that OS-controlled on-line memory compression is a symmetric application, i.e., a memory page is decompressed exactly once every time it is compressed. Therefore, the overall, symmetric, performance of a compression algorithm is the critical performance metric. Overall, PBPM achieves a $200 \%$ speedup over LZO and LZRW. Yet the compression ratio achieved by PBPM is comparable with that of LZO and LZRW. We believe that PBPM is especially suitable for on-line memory compression because of its extremely fast symmetric compression and good compression ratio. 


\begin{tabular}{|l|}
\hline \multicolumn{1}{|c|}{ Benchmark Description } \\
\hline Adpcm: Speech compression \\
Source: MediaBench \\
Data size: $24 \mathrm{~KB}$ \\
Code size: $4 \mathrm{~KB}$ \\
\hline Jpeg: Image encoding \\
Source: MediaBench \\
Data size: $176 \mathrm{~KB}$ \\
Code size: $72 \mathrm{~KB}$ \\
\hline Mpeg2: Video CODEC \\
Source: MediaBench \\
Data size: $416 \mathrm{~KB}$ \\
Code size: $48 \mathrm{~KB}$ \\
\hline $\begin{array}{l}\text { Matrix Mul.: } 512 \text { by } 512 \text { matrix } \\
\text { multiplication } \\
\text { Data size: } 2948 \mathrm{~KB} \\
\text { Code size: } 4 \mathrm{~KB}\end{array}$ \\
\hline
\end{tabular}

\begin{tabular}{|c|c|c|c|c|c|c|c|c|c|c|c|c|}
\hline \multirow{2}{*}{$\begin{array}{l}\text { RAM } \\
\text { (MB) }\end{array}$} & \multicolumn{3}{|c|}{ Adpcm } & \multicolumn{3}{|c|}{ Jpeg } & \multicolumn{3}{|c|}{ Mpeg2 } & \multicolumn{3}{|c|}{ Matrix Mul. } \\
\hline & w.o. & LZO & PBPM & w.o. & $\mathrm{LZO}$ & PBPM & w.o. & LZO & PBPM & w.o. & LZO & PBPM \\
\hline \multicolumn{13}{|c|}{ Execution Time (s) } \\
\hline 8 & 4.83 & 1.69 & 1.43 & 0.71 & 0.26 & 0.23 & 79.35 & 80.30 & 77.96 & n.a & 39.26 & 38.68 \\
\hline 9 & 3.69 & 1.35 & 1.26 & 0.44 & 0.21 & 0.21 & 76.80 & 76.83 & 74.04 & n.a & 37.40 & 38.24 \\
\hline 10 & 1.41 & 1.34 & 1.36 & 0.23 & 0.21 & 0.21 & 79.06 & 76.93 & 75.32 & 59.11 & 39.56 & 37.18 \\
\hline 11 & 1.37 & 1.40 & 1.40 & 0.26 & 0.25 & 0.21 & 80.57 & 76.81 & 76.83 & 44.44 & 38.42 & 42.65 \\
\hline 12 & 1.37 & 1.31 & 1.32 & 0.24 & 0.21 & 0.19 & 76.79 & 76.94 & 76.95 & 41.72 & 38.73 & 43.96 \\
\hline 20 & 1.31 & 1.30 & 1.30 & 0.23 & 0.21 & 0.22 & 76.60 & 76.77 & 76.76 & 43.02 & 41.41 & 42.97 \\
\hline \multicolumn{13}{|c|}{ Power Consumption (W) } \\
\hline 8 & 2.13 & 2.13 & 2.13 & 2.15 & 2.16 & 2.15 & 2.41 & 2.41 & 2.51 & n.a & 2.26 & 2.29 \\
\hline 9 & 2.10 & 2.10 & 2.13 & 2.15 & 2.02 & 2.07 & 2.41 & 2.40 & 2.50 & n.a & 2.26 & 2.29 \\
\hline 10 & 2.09 & 2.10 & 2.09 & 2.00 & 1.99 & 2.04 & 2.39 & 2.40 & 2.48 & 2.24 & 2.25 & 2.29 \\
\hline 11 & 2.12 & 2.09 & 2.13 & 2.05 & 2.04 & 2.07 & 2.40 & 2.40 & 2.50 & 2.26 & 2.25 & 2.29 \\
\hline 12 & 2.09 & 2.13 & 2.11 & 2.03 & 2.05 & 2.10 & 2.40 & 2.41 & 2.55 & 2.25 & 2.25 & 2.29 \\
\hline 20 & 2.11 & 2.09 & 2.18 & 2.15 & 2.02 & 2.24 & 2.42 & 2.43 & 2.57 & 2.28 & 2.27 & 2.29 \\
\hline \multicolumn{13}{|c|}{ Energy Consumption $(\mathrm{J})$} \\
\hline 8 & 10.34 & 3.60 & 3.04 & 1.51 & 0.56 & 0.49 & 190.99 & 193.42 & 195.71 & $\mathrm{n}$. & 88.74 & 88.62 \\
\hline 9 & 7.75 & 2.84 & 2.68 & 0.94 & 0.42 & 0.43 & 185.38 & 184.55 & 185.10 & n.a & 84.70 & 87.64 \\
\hline 10 & 2.94 & 2.79 & 2.85 & 0.47 & 0.42 & 0.42 & 188.62 & 184.34 & 186.42 & 131.05 & 88.99 & 85.01 \\
\hline 11 & 2.89 & 2.93 & 2.97 & 0.54 & 0.52 & 0.44 & 193.10 & 184.69 & 191.94 & 100.01 & 86.38 & 97.79 \\
\hline 12 & 2.86 & 2.79 & 2.79 & 0.49 & 0.43 & 0.41 & 184.45 & 185.74 & 196.33 & 93.65 & 86.94 & 100.81 \\
\hline 20 & 2.75 & 2.72 & 2.82 & 0.48 & 0.43 & 0.49 & 185.72 & 186.56 & 197.26 & 98.27 & 94.07 & 98.39 \\
\hline
\end{tabular}

Figure 4: Overall performance of CRAMES

\subsection{Effectiveness of adaptive memory management}

In order to determine the effectiveness of adaptive memory management in providing more memory to applications under significant memory pressure, we designed the following experiments. We wrote a 'memeater' program that continuously requests $1 \mathrm{MB}$ of memory at a time and fills the allocated memory with random numbers (including zero runs with similar frequency to that observed in real swap traces), until an allocation request fails. Memeater was then executed on Zaurus under three different system settings: without using CRAMES (none), using CRAMES without adaptive memory management (CRAMES), and using CRAMES with adaptive memory management (A-CRAMES). Figure 3 presents the total memory allocated and average execution times under the three system settings (memeater was executed multiple times under each setting). Without CRAMES, the system was only able to provide $16 \mathrm{MB}$ of memory to memeater. With CRAMES, $33 \mathrm{MB}$ of memory were provided and the execution time was proportional to the amount of memory allocated, i.e., no delay was observed. Furthermore, when adaptive memory management is enabled (ACRAMES), $38 \mathrm{MB}$ of memory were allocated with no additional cost. These results support our claim in Section 5 that A-CRAMES helps to prevent on-line data compression deadlock.

\subsection{Overall performance of the improved CRAMES}

Embedded system with high-performance on-line memory compression can be designed with less RAM and still support desired applications. In order to evaluate the impact of using CRAMES to reduce physical RAM, we artificially constrained the memory size of a Zaurus with a kernel module that permanently reserves a certain amount of physical memory. The memory allocated to a kernel module cannot be swapped out and therefore is not compressed by CRAMES. This guarantees the fairness of our comparison. With reduced physical RAM, we measured and compared the run times, power consumptions, and energy consumptions of four batch benchmarks, i.e., three applications from MediaBench [11] (Adpcm, Jpeg, Mpeg2) and a 512 by 512 matrix multiplication application. Figure 4 shows execution times, power consumptions, and energy consumptions of benchmarks running without compression, with LZO compression, and with PBPM compression under different memory constraints. Note that adaptive memory management was enabled in both LZO and PBPM compression to ensure fair comparison. In our experiments, each benchmark was executed multiple times; the average results are reported.

As shown in Figure 4, when system RAM was reduced to $8 \mathrm{MB}$, without CRAMES, all benchmarks suffered from significant performance degradation; the 512 by 512 matrix multiplication couldn't even execute due to memory constraints. However, with the help of CRAMES, all benchmarks were able to execute with only slight performance and energy consumption penalties. Compared with the base case in which system RAM is $20 \mathrm{MB}$ and CRAMES is not used, PBPM compression results in an average performance penalty of $2.1 \%$ and a worst-case performance penalty of $9.2 \%$. This rep- resents a substantial improvement over LZO, for which the average performance penalty is $9.5 \%$ and the worst-case performance penalty can be as high as $29 \%$.

\section{Conclusions and acknowledgments}

High-performance OS controlled memory compression can assist embedded system designers to optimize hardware design for typical software memory requirements while also supporting (sets of) applications with larger data sets. In this paper, we proposed and evaluated a fast software-based compression algorithm for use in this application. This algorithm provides comparable compression ratios to existing algorithms used in on-line memory compression with significantly better symmetric performance. We also presented an adaptive compressed memory management scheme to prevent on-line memory compression deadlock, permitting reliable and efficient on-line memory compression for a wide range of applications. Experimental results indicate that the improved CRAMES allows applications to execute with only slight penalties even when system RAM is reduced to $40 \%$ of its original size.

We would like to acknowledge Dr. Srimat Chakradhar of NEC Laboratories America for his support and technical advice. We would also like to acknowledge Hui Ding of Northwestern University for his suggestions on efficiently implementing PBPM.

\section{References}

[1] L. Yang, et al., "CRAMES: Compressed RAM for embedded systems," in Proc. Int. Conf. Hardware/Software Codesign and System Synthesis, Sept. 2005.

[2] J. Ziv and A. Lempel, "A universal algorithm for sequential data compression," IEEE Transactions on Information Theory, vol. 23, no. 3, pp. 337-343, 1977.

[3] B. Tremaine, et al., "IBM memory expansion technology," IBM Journal of Research and Development, vol. 45, no. 2, Mar. 2001.

[4] H. Lekatsas, J. Henkel, and W. Wolf, "Code compression for low power embedded system design," in Proc. Design Automation Conf., June 2000, pp. 294-299.

[5] F. Douglis, "The compression cache: Using on-line compression to extend physical memory," in Proc. USENIX Conf., Jan. 1993.

[6] P. R. Wilson, S. F. Kaplan, and Y. Smaragdakis, "The case for compressed caching in virtual memory systems," in Proc. USENIX Conf., 1999, pp. 101-116.

[7] L. Rizzo, "A very fast algorithm for RAM compression," Operating Systems Review, vol. 31, no. 2, pp. 36-45, Apr. 1997.

[8] I. C. Tuduce and T. Gross, "Adaptive main memory compression," in Proc. USENIX Conf., Apr. 2005.

[9] M. Kjelso, M. Gooch, and S. Jones, "Performance evaluation of computer architectures with main memory data compression," in $J$. Systems Architecture, vol. 45, 1999, pp. 571-590.

[10] "LZO real-time data compression library," http://www.oberhumer.com/opensource/lzo.

[11] C. Lee, M. Potkonjak, and W. H. M. Smith, "Mediabench: A tool for evaluating and synthesizing multimedia and communications systems," http://cares.icsl.ucla.edu/MediaBench. 